\title{
Observation of off-axis directional beaming via subwavelength asymmetric metallic gratings
}

\author{
H Caglayan ${ }^{1}$, I Bulu ${ }^{2}$ and E Ozbay ${ }^{1}$ \\ ${ }^{1}$ Nanotechnology Research Center-NANOTAM, Department of Physics, Department of Electrical and \\ Electronics Engineering, Bilkent University, Bilkent, 06800 Ankara, Turkey
}

The transmission of electromagnetic (EM) waves through a singlesubwavelengthaperturehasbeenstudiedformanyyears. As defined in the standard diffraction theory by Bethe [1] in 1944, EM waves that transmit through a subwavelength aperturearefullydiffractedinalldirections. Thisdisadvantage is the main problem in controlling light. Hence, there have been many studies that have aimed at solving this problem. In 2002, Lezec et al [2] showed that it is possible to obtain enhanced as well as directional beaming from subwavelength apertures via the excitation of surface plasmons on corrugated metallic surfaces. Since the reporting of directional beaming through subwavelength apertures, this phenomenon has been intensively researched in its theoretical and experimental aspects [3-7]. Enhanced and directional beaming studies are not just limited to metallic gratings. There is much research activity being conducted on the beaming of light using photonic crystals [8-10]. However, all these studies specifically investigated on-axis beaming.

Changing the beaming angle is another important issue. Therefore, only if we can control both the beaming and the beaming angle can we in turn fully solve the problem of diffraction from a subwavelength aperture. Recently, Kim et al [11] proposed a method for changing the beaming angle, and Lin et al [12] showed that it is possible to obtain off-axis directional beaming at optical frequencies using asymmetric dielectric gratings. However, to our knowledge, there has not been any scientific work that demonstrates off-axis directional beaming by using metallic gratings in the microwave regime. In this work, we investigated the steering of beaming via a subwavelength metallic aperture with asymmetric surface gratings. By using this new structure, we were able to change the beaming angle and attain off-axis directional beaming.

Surface plasmons are the collective excitation of electrons at the surface of a conductor in the longitudinal direction. Since surface plasmon modes have longer wave vectors than the light waves of the same energy, EM radiation does not interact with the surface plasmon modes of a smooth metal surface. However, when the metal surface surrounding the subwavelength aperture is corrugated, the incident light can couple to surface waves that mimic surface plasmons [2]. The metallic structures that were used in this work have a subwavelength slit $(\lambda / 10)$ at the centre. The details of the 
structures are shown in figure 1. The grating period on the input side is $16 \mathrm{~mm}$ for all three structures. At microwave

E-mail: caglay@bilkent.edu.tr

Received 4 August 2008, in final form 18 November 2008

Published 28 January 2009

Online at stacks.iop.org/JPhysD/42/045105

\section{Abstract}

It is possible to obtain enhanced and directional beams using subwavelength metallic structures. However, the enhanced beams throughout such structures are only directed

towards the propagation direction. In this study, we design the output surface gratings asymmetrically in order to steer the beaming angle. We use a metallic structure with a subwavelength slit $(\lambda / 10)$ and grating periods of $14 \mathrm{~mm}$ and $22 \mathrm{~mm}$ on different sides of the output surface. We demonstrate off-axis directional beaming in the microwave regime with an FWHM of $10^{\circ}$ with a beaming angle of $15^{\circ}$. The beaming angle can be changed by arranging the grating periods of the output surface of the metallic structure.

(Some figures in this article are in colour only in the electronic version)
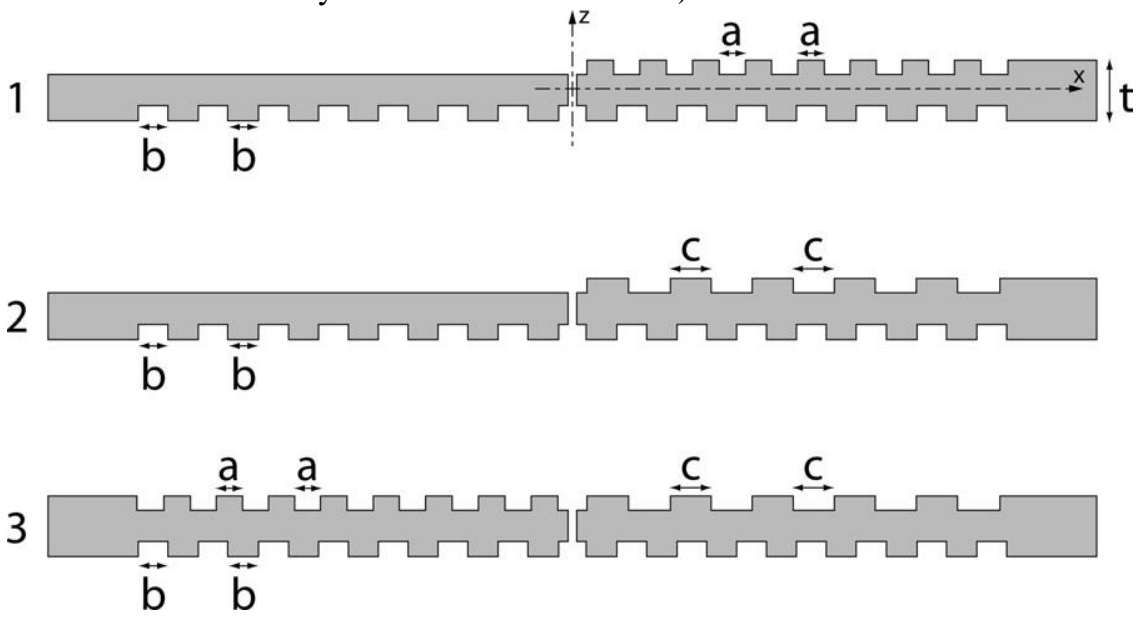
Figure 1. The structures used in this work were $\mathrm{a}=7 \mathrm{~mm}, \mathrm{~b}=8 \mathrm{~mm}, \mathrm{c}=11 \mathrm{~mm}$ and $\mathrm{t}=16 \mathrm{~mm}$. The slit widths were $2 \mathrm{~mm}$ and the grating

heights were $4 \mathrm{~mm}$. All of them had the same input surface grating period of $16 \mathrm{~mm}$ in order to couple the 'spoof' surface plasmons.

frequencies, surface plasmons are too weakly bound to the surface; therefore the, EM response from these structures is governed by the surface modes [13] and they are frequently referred to as 'spoof' surface plasmons or 'designer' surface plasmons. The gratings on the input side of the structures stand for the coupling of surface modes. The grating period of $16 \mathrm{mmexecutesaresonanceataround} 14.5 \mathrm{GHz}(20.7 \mathrm{~mm})[4]$. On the one hand, the output surface is responsible for the beaming effect $[2,14]$. When the subwavelength aperture is illuminated on the side of the grooved surface (no grooves on the exit surface), the beam diffracts in all directions. However, a beaming effect occurs when the sample is illuminated so that the output surface is grooved. The emitted EM waves are confined to a narrow spatial region when the output surface of the subwavelength aperture was surrounded by periodic gratingssincethesurface-wavemomentumandthemomentum of the corrugation around the subwavelength aperture limit the allowed range of momentum of the reradiated EM waves.

The beam is directed on the axis of the propagation direction, if the subwavelength aperture has symmetric grating periods on different sides of the output surface. However, it is possible to steer the beaming angle by using asymmetric grating periods. In order to optimize the grating periods, we first investigated the structures with gratings only on one side. We calculated the angular distribution of the transmission for a structure with a grating period of $14 \mathrm{~mm}$ on one side of the output surface (Sample 1). In this study, calculations were performed by using the commercial software (FULLWAVE), which uses the finite difference time domain (FDTD) method. In the simulation, an EM wave with an $\mathrm{E}$ field that was polarized in the $\mathrm{x}$ direction is incident from the bottom. After thefieldpassesthroughtheaperture, itemitsinthe+zdirection. The EM waves throughout Sample 1 are mostly directed to the negative side (we defined the right side of the $90^{\circ}$ as positive and the left side as negative) at $14.5 \mathrm{GHz}$ (figure $2(\mathrm{a})$ ).

The measurements were performed in the microwave spectrum at $14.5 \mathrm{GHz}$. The experimental setup consists of a Hewlett Packard 8510C network analyzer and two standardgain horn antennas in order to measure the transmission amplitude. Radiation is normally incident upon the

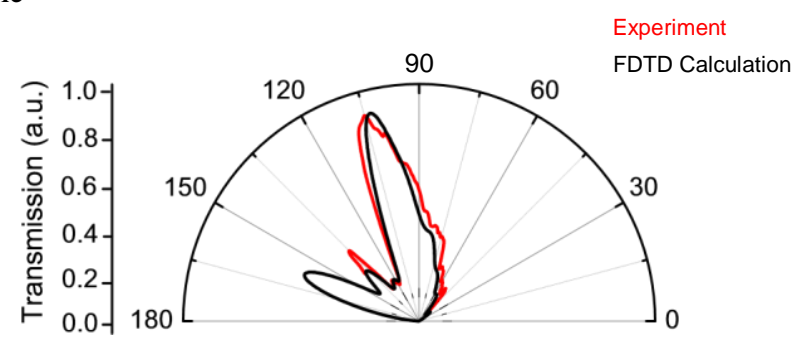

(a)
Experiment

FDTD Calculation

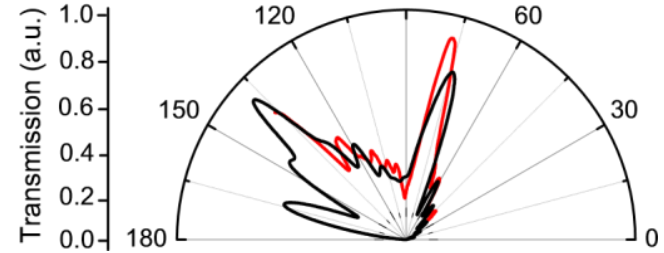

(b)

Figure 2. The beaming angle is steered for structures with grating periods only on one side of the output surface. The beam is mostly directed to the negative side for (a) Sample 1 and positive side for (b) Sample 2 at $14.5 \mathrm{GHz}(20.7 \mathrm{~mm})$.

sample from $15 \mathrm{~cm}$ by the source antenna. We have chosen $\mathrm{p}$ polarization (TM polarization). The receiver antenna was placed $100 \mathrm{~cm}(\approx 50 \lambda)$ away from the sample's back face and wasconnectedtoarotatingarminordertomeasuretheangular dependence of the radiation. We observed that most of the EM waves radiated towards the side without grating at around $-15^{\circ}$ for Sample 1 at $14.5 \mathrm{GHz}$, which corresponds to the spoof plasmon resonance frequency (figure 2). This is also in agreement with the FDTD calculations.

AlthoughtheEMwavesthroughoutSample1 weremostly directed towards $-15^{\circ}$, the beam was not collimated to the narrow range. In order to obtain off-axis directional beaming we need to arrange the other side of the sample with a period that is different from $14 \mathrm{~mm}$. We optimized this period by FDTD calculations to where the structure emits EM waves to the positive side. The calculated transmission for the structure

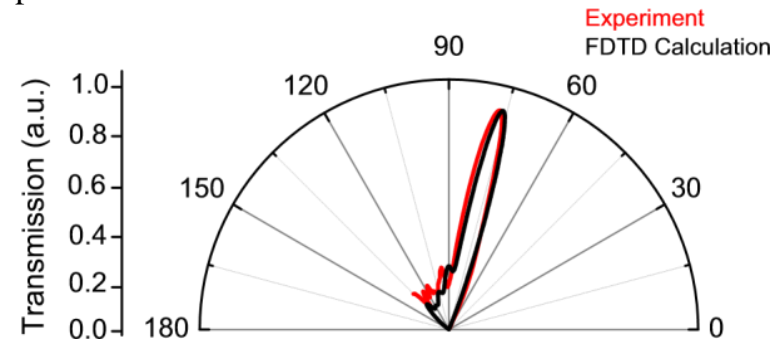




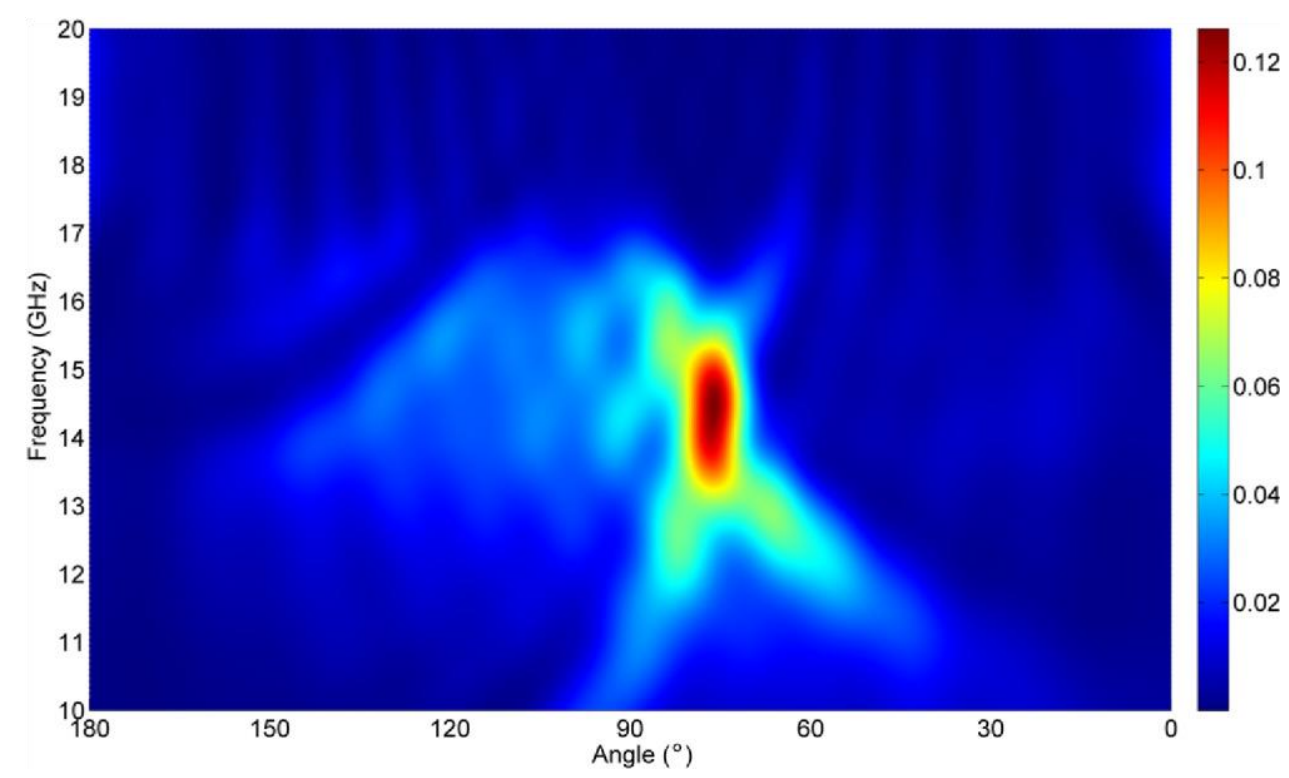

Figure 3. The calculated transmission throughout Sample 3 shows that off-axis and directional beaming is possible by using metallic asymmetric gratings on the output surface at resonance frequency $(14.5 \mathrm{GHz})$.

Figure 4. By use of a metallic structure with a subwavelength aperture at the centre and the grating periods of $14 \mathrm{~mm}$ and $22 \mathrm{~mm}$ on the different sides of the output surface, we observed off-axis directional beaming with an FWHM of $10^{\circ}$ and the beaming angle of $15^{\circ}$. It is possible to steer the beaming angle by arranging the grating periods of the output surface of the metallic structure.

with a grating period of $22 \mathrm{~mm}$ (Sample 2) shows that most of the emitted EM waves were directed towards the positive side. In the measurements of our second sample, we observed a beam at $15^{\circ}$ on the positive side (figure 2(b)). This case is the exact opposite of Sample 1.

It is possible to steer the beam by only using Sample 1 or 2. However, the beaming is not as clear for them. In order to obtain off-axis as well as directional beaming, we combined these two structures (Sample 3). The combination of these gratings will provide a directional yet off-axis beam through the positive side. The calculated transmission between 10 and $20 \mathrm{GHz}$ from Sample 3 shows that at the resonance frequency $(14.5 \mathrm{GHz})$ the emitted EM waves were directed towards the positive side (figure 3). Meanwhile, we measured the angular distribution of the EM waves that were transmitted through Sample 3 with asymmetric grating periods on different sides. We observed off-axis directional beaming with a beaming angle of $15^{\circ}$ and a full-width half-maximum (FWHM) of $10^{\circ}$ (figure 4 ). $12 \%$ of the power is transmitted from the metallic structure with a subwavelength aperture at the resonance frequency. This result is in good agreement with the FDTD calculations. Since the beaming angle only depends on the grating periods of the output side, this angle can be changed easily by arranging the output grating periods asymmetrically. We have also presented the FDTD mode pattern showing the coupling between the top and bottom surfaces at the resonance frequency (figure 5).
This phenomenon can be explained by the use of the dispersion relation of the surface bound states. The dispersion relation of the surface modes is given as [13]

$$
\frac{\sqrt{k_{x}^{2}-k_{0}^{2}}}{k_{\mathrm{o}}^{2}}=\frac{a}{d} \tan \left(k_{0} h\right),
$$

where $\mathrm{h}$ is the depth of the grooves, $\mathrm{a}$ is the groove width and $\mathrm{d}$ isthegrooveperiod. Infigure6weplotthedispersionrelations for groove periods $14 \mathrm{~mm}$ and $22 \mathrm{~mm}$. This dispersion relation is related to the beaming angle of the off-axis directional beaming appearing. The angle between the wavevector of the surface mode and the incident EM wave gives the beaming angle. The calculated beaming angles using this dispersion relation are $14^{\circ}$ and $16^{\circ}$ for metallic structures with groove periods of $14 \mathrm{~mm}$ and $22 \mathrm{~mm}$, respectively. These results are in agreement with FDTD calculations. It is also possible to describe the angular width and the propagation direction with grating coupler formulae.

In conclusion, we were able to change the beaming angle of the directional beaming by using metallic asymmetric gratings on the output surface. By using a metallic structure with a subwavelength aperture at the centre and the grating periods of $14 \mathrm{~mm}$ and $22 \mathrm{~mm}$ on the different sides of the output surface, we observed off-axis directional beaming with an FWHM of $10^{\circ}$. We showed that the beaming angle is $15^{\circ}$. Furthermore, these results are in good agreement with the FDTD calculations. Our results show that it is possible to steer the beaming angle by arranging the grating periods of the output surface of the metallic structure. 

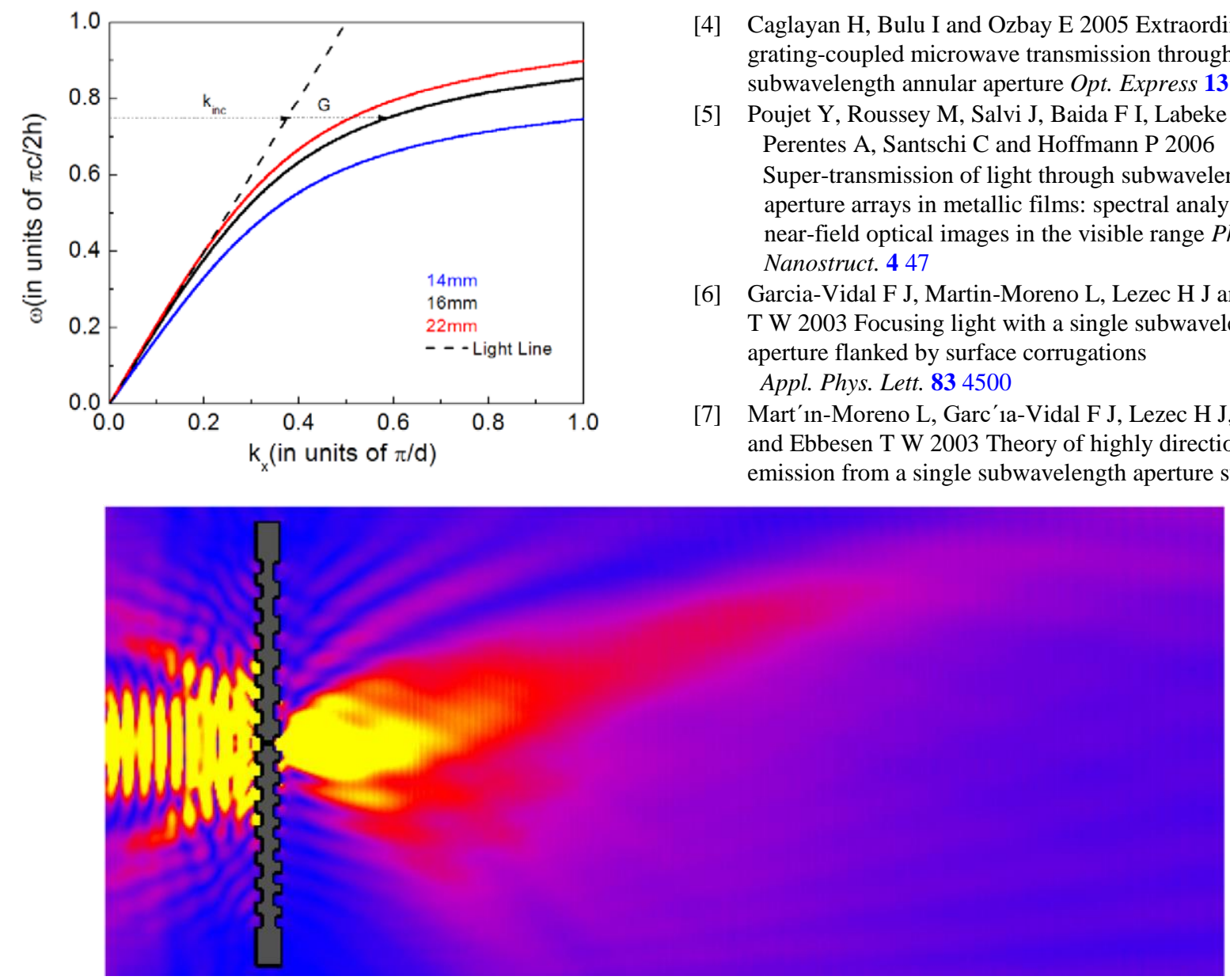

[4] Caglayan H, Bulu I and Ozbay E 2005 Extraordinary grating-coupled microwave transmission through a subwavelength annular aperture Opt. Express 13 1666-71

[5] Poujet Y, Roussey M, Salvi J, Baida F I, Labeke D, Perentes A, Santschi C and Hoffmann P 2006 Super-transmission of light through subwavelength annular aperture arrays in metallic films: spectral analysis and near-field optical images in the visible range Photon. Nanostruct. 447

[6] Garcia-Vidal F J, Martin-Moreno L, Lezec H J and Ebbesen T W 2003 Focusing light with a single subwavelength aperture flanked by surface corrugations Appl. Phys. Lett. 834500

[7] Mart'in-Moreno L, Garc'ia-Vidal F J, Lezec H J, Degiron A and Ebbesen T W 2003 Theory of highly directional emission from a single subwavelength aperture surrounded

Figure 5. FDTD mode pattern showing the coupling between the top and bottom surfaces at the resonance frequency.

Figure 6. The dispersion relation of the surface modes supported by metallic structures with grooves of periods $14 \mathrm{~mm}$ (blue line), $16 \mathrm{~mm}$ (black line) and $22 \mathrm{~mm}$ (red line). The light line is shown with a dashed line. (Colour online.)

\section{Acknowledgments}

This work is supported by the European Union under the projects EU-METAMORPHOSE, EU-PHOREMOST, EUPHOME, EU-ECONAM and TUBITAK under the Project Numbers 105E066, 105A005, 106E198 and 106A017. One of the authors (EO) also acknowledges partial support from the Turkish Academy of Sciences.

\section{References}

[1] Bethe H A 1944 Theory of diffraction by small holes Phys. Rev. 66163182

[2] Lezec H J, Degiron A, Devaux E, Linke R A, Martin-Moreno L, Garcia-Vidal F J and Ebbesen T W 2002 Beaming light from a subwavelength aperture Science $297820-2$

[3] Yu L B, Lin D Z, Chen Y C, Chang Y C, Huang K T, Liaw J $\mathrm{W}$,

Yeh J T, Liu J M, Yeh C S and Lee C K 2005 Physical origin of directional beaming emitted from a subwavelength slit Phys. Rev. B 71041405 by surface corrugations Phys. Rev. Lett. 90167401

[8] Moreno E, Garcia-Vidal F J and Martin-Moreno L 2004 Enhanced transmission and beaming of light via photonic crystal surface modes Phys. Rev. B 69121402

[9] Bulu I, Caglayan H and Ozbay E 2005 Beaming of light and enhanced transmission via surface modes of photonic crystals Opt. Lett. 303078

[10] Kramper P, Agio M, Soukoulis C M, Birner A, Muller F," Wehrspohn R B, Gosele U and Sandoghdar V 2004 Highly" directional emission from photonic crystal waveguides of subwavelength width Phys. Rev. Lett. 92113903

[11] Kim S, Kim H, Lim Y and Lee B 2007 Off-axis directional beaming of optical field diffracted by a single subwavelength metal slit with asymmetric dielectric surface gratings Appl. Phys. Lett. 90051113

[12] Lin D Z, Cheng T D, Chang C K, Yeh J T, Liu J M, Yeh C S and Lee C K 2007 Directional light beaming control by a subwavelength asymmetric surface structure Opt. Express 15 2585-91

[13] Garcia-Vidal F J, Martin-Moreno L and Pendry J B 2005 Surface with holes in them: new plasmonic metamaterials J. Opt. A: Pure Appl. Opt. 7 S97-S101

[14] Caglayan H, Bulu I and Ozbay E 2006 Plasmonic structures with extraordinary transmission and highly directional beaming properties IEEE Microw. Opt. Technol. Lett. 482491 